\title{
Corrosion Resistance of HastelloyC Coatings Formed by an Improved HVOF Thermal Spraying Process
}

\author{
Jin Kawakita, Seiji Kuroda, Takeshi Fukushima and Toshiaki Kodama \\ National Institute for Materials Science, Tsukuba 305-0047, Japan
}

The corrosion resistance of coatings obtained by an improved HVOF spraying method was evaluated by various electrochemical techniques. This spraying system could increase the flight velocity of sprayed particles and suppress their oxidation simultaneously by attachment of a gas shroud, employing nitrogen gas at a large flow rate. The process brought about attainment of both smaller porosity and lower oxide content of the resulting coatings. Consequently, the resistance of HVOF sprayed coatings against seawater corrosion was improved considerably.

(Received July 22, 2002; Accepted November 8, 2002)

Keywords: high velocity oxy-fuel (HVOF), HastelloyC, gas shroud, corrosion resistance, seawater

\section{Introduction}

One of our national research project named as Ultra Steel for the 21st Century (STX-21) is to coat the offshore structural steels with anti-corrosion alloys by the thermal spray technique. Targets of the spray coating are to protect the tidal and the splash zones of these structures from severely corrosive environments. In such environments, significantly high corrosion resistance is essential. For this reason, a nickel base alloy, HastelloyC, was selected as the coating material because of its high resistance against both crevice and pitting corrosion as well as its appropriate melting point for the following spraying method. The high velocity oxy-fuel (HVOF) spraying was adopted because this can deposit a denser coating with less oxide than those by conventional methods such as plasma spraying.

HastelloyC coatings prepared according to the standard condition recommended by the apparatus manufacturer had low open-porosity below the detection limit by mercury intrusion porosimetry. ${ }^{1,2)}$ The HastelloyC coating itself, immersed in artificial seawater for 3 days, showed no evidence of corrosion damage while small rusts with no easy visibility appeared on the surface of the same type of coating deposited on a mild steel, SS400. This result indicated the existence of a slight amount of through-pores, along which seawater could permeate the coating, leading to the formation of a galvanic cell at the interface between the electrochemically noble coating and the less-noble substrate. Then, various spray conditions were examined to establish the guideline for decrease in through-porosity of the coating down to zero. At the same time, a method for detection of through-pores was devised and developed, which had the higher sensitivity by two orders of magnitude than mercury intrusion porosimetry. ${ }^{3,4)}$ By raising the flame energy upon spraying, through-porosity of the HastelloyC coating was found to attain zero because no through-pores were detected even by using the above-mentioned. However, the higher flame energy brought about the higher oxide content of the coating, leading to the decrease in corrosion resistance of the coating itself.

In order to realize the compatibility of both the high flame energy and the less oxidized particles during thermal spraying, the mechanism that flying particles were surrounded by an inert gas was devised. ${ }^{5)}$ A pipe was attached to one end of the barrel of a commercial HVOF gun, as illustrated schematically in Fig. 1. The inert gas was injected from both the ends of this pipe. This mechanism was called as the "gas shroud mechanism" or the "shroud mechanism" below. It can accelerate and simultaneously cool the flying particles during spraying. When AISI 316L stainless steel (SUS316L) was used as the feed stock powder, the average flight velocity was over $750 \mathrm{~m} \cdot \mathrm{s}^{-1}$ and their molten fraction was below 40 mass\%. ${ }^{5)}$ As a result, significantly denser and lessoxidized coatings were obtained so that this shroud mechanism allows the flying particles to avoid encountering atmosphere in the longer distance than as usual. Although some

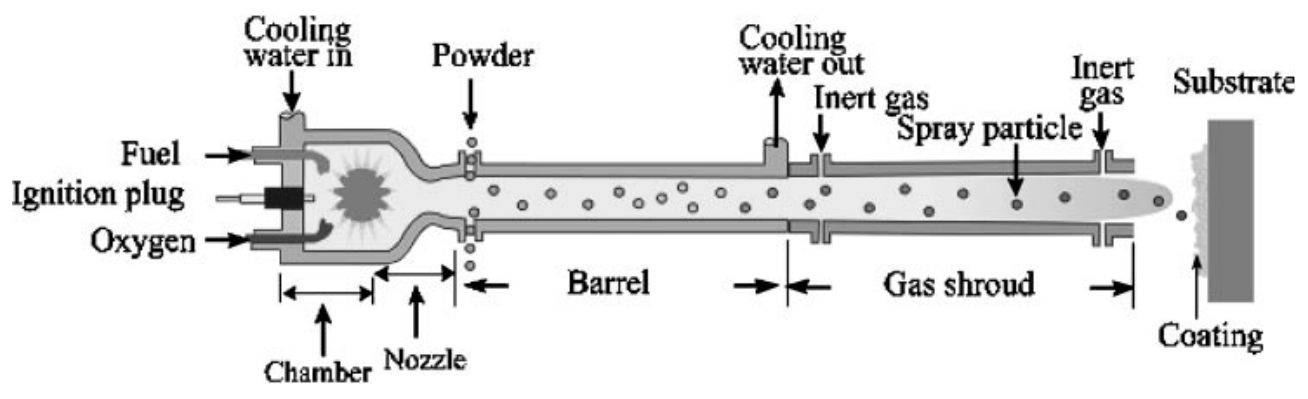

Fig. 1 Schematic illustration of gas shroud attachment with HVOF spray gun. 
research groups studied adoption of such a gas shroud, ${ }^{6-8)}$ their systems were different from ours with respect to the spraying apparatus, the spraying material, and especially the shroud mechanism.

In this paper, the HastelloyC coating deposited by HVOF spraying with the gas shroud was studied in terms of its physical and chemical properties such as the porosity and the chemical composition. Corrosion resistance of this coating was examined with several electrochemical techniques. In addition, the above-mentioned characteristics of this coating were compared with those of other coatings obtained before.

\section{Experimental}

Coating on JIS SS400 substrate was carried out by HVOF spraying (TAFA JP-5000) with kerosene as the fuel. The primary spray condition is listed in Table 1 . The substrate of $t 2 \mathrm{~mm} \times 50 \mathrm{~mm} \times 100 \mathrm{~mm}$ was blasted using alumina grits and degreased ultrasonically in acetone before spraying. The feedstock is the powders of HastelloyC and SUS316L. SUS316L was used as the reference to be compared because it is well known to be a corrosion resistant material. Their chemical compositions are presented in Table 2. A nitrogen gas was injected into the shroud at the flow rates of $2.5 \mathrm{~m}^{3} \cdot \mathrm{min}^{-1}$ from upstream and at $0.3 \mathrm{~m}^{3} \cdot \mathrm{min}^{-1}$ from downstream. In this paper, a typical condition recommended by the manufacturer was called as "standard" and the condition with the shroud attachment was called as "onshroud".

The oxygen content and the open porosity of the coatings were obtained by the inert-gas fusion method and by mercury intrusion porosimetry (Micromeritics Autopore II 9220), respectively. Their through-porosity was evaluated by Inductively Coupled Plasma Atomic Emission Spectrometry (ICP-AES, Seiko Instruments Inc. SPS 3000) of the ion dissolved from the substrate while the coated specimen was

Table 1 Spray conditions.

\begin{tabular}{lcc}
\hline \multicolumn{1}{c}{ Parameter } & Unit & Range \\
\hline Fuel flow rate & $\mathrm{dm}^{3} \cdot \mathrm{min}^{-1}$ & $0.25-0.49$ \\
Oxygen flow rate & $\mathrm{dm}^{3} \cdot \mathrm{min}^{-1}$ & $760-1080$ \\
Combustion pressure & $\mathrm{MPa}$ & $0.43-0.86$ \\
Fuel/oxygen ratio*1 & - & $0.7-1.2$ \\
Barrel length & $\mathrm{mm}$ & 102,204 \\
Powder feed rate & $\mathrm{g} \cdot \mathrm{min}^{-1}$ & 60 \\
Torch velocity & $\mathrm{mm} \cdot \mathrm{s}^{-1}$ & 700 \\
Spray distance & $\mathrm{mm}$ & 482 \\
Powder feed gas & - & Nitrogen $\left(\mathrm{N}_{2}\right)$ \\
Film thickness. & $\mu \mathrm{m}$ & $50-400$ \\
\hline *1 1.0 corresponds to stoichiometric mixture ratio. & \\
$* 2 \quad$ From exit of combustion chamber. &
\end{tabular}

immersed in $0.5 \mathrm{~mol} \cdot \mathrm{dm}^{-3}$ of $\mathrm{HCl}^{3,4)}$

Corrosion resistance of the coated specimen was evaluated by the polarization and the alternating current (AC) impedance methods. The detailed procedure was described elsewhere. ${ }^{9)}$ The sample electrode was prepared in the following sequence: 1) the coated specimen was cut into pieces of $2.5 \mathrm{~cm}$ square and cleaned ultrasonically in acetone and ion-exchanged water, repeatedly, 2) AISI 304 stainless wire was connected to the back surface of the substrate plate by spot welding, and 3) the sprayed area of $2 \mathrm{~cm}^{2}$ was left exposed and the rest of the specimen surface was insulated with silicone resin. The reference electrode was the $\mathrm{Ag} / \mathrm{AgCl}$ electrode in the saturated $\mathrm{KCl}$ solution. The electrolyte was artificial seawater of $\mathrm{pH} 8.3$ at $300 \mathrm{~K}$. The salt bridge of agar containing $\mathrm{KCl}$ was used to avoid mixing the artificial seawater and the $\mathrm{KCl}$ solution. Polarization curve was obtained at the scanning rate of $10 \mathrm{mV} \cdot \mathrm{s}^{-1}$ using a potentiostat with a function generator (Hokuto Denko HAB-151). The sample electrode was immersed in the electrolyte for 24 hours to reach the steady state. The counter electrode was a platinum plate with a dimension of $t 0.2 \mathrm{~mm} \times 100 \mathrm{~mm} \times$ $100 \mathrm{~mm}$, and continous nitrogen bubbling was carried out for deaeration of the electrolyte. From the impedance measurement, the polarization resistance of the sample was determined by subtracting one impedance value at the frequency of $10 \mathrm{kHz}$ from another at $100 \mathrm{mHz}$ with the corrosion monitor (Riken Denshi Model CT-5). In this measurement, the same type of sample electrodes were used for both the working and counter electrodes, and continuous air bubbling was carried out for aeration of the electrolyte. This polarization resistance was monitored every 10 minutes for 3 days in addition to the corrosion potential. For comparison, a blasted plate of HastelloyC276 was used because of the increase in actual surface area.

\section{Results and Discussion}

In general, the open-porosity of the HVOF sprayed coatings is inversely related to their oxygen content, as shown in Fig. 2. Some plots concerning a kind of material in this figure indicates values obtained under different spray conditions. This relation appears to be a trade-off, and both properties were difficult to be improved (i.e. decrease) together in the HVOF spray process. However, the gas shroud overcame this problem. As for the HastelloyC coating prepared with the shroud attachment, the open-porosity below 0.1 vol\% was attained accompanying with 0.2 mass $\%$ of oxygen content. Such a small porosity was not detectable by mercury intrusion porosimetry, and then it was evaluated precisely by our method, as mentioned above. Figure 3 shows that the dissolved Fe ion derived from the SS400 substrate could be detected in $0.5 \mathrm{~mol} \cdot \mathrm{dm}^{-3}$ of $\mathrm{HCl}$ even for the HastelloyC coating with open-porosity below 0.1 vol\% and

Table 2 Chemical compositions of feedstock powders.

\begin{tabular}{cl}
\hline \multicolumn{1}{c}{ Material } & \multicolumn{1}{c}{ Composition (mass\%) } \\
\hline HastelloyC (TAFA1286F) & Ni bal, Mo 16.95, Cr 16.57, Fe 6.2, W 4.5, Mn 0.72, Co 0.31, Si 0.73 \\
SUS316L (TAFA 1236F) & Fe bal, Cr 16.8, Ni 10.8, Mo 2.05, N 0.131, O 0.026 \\
\hline
\end{tabular}




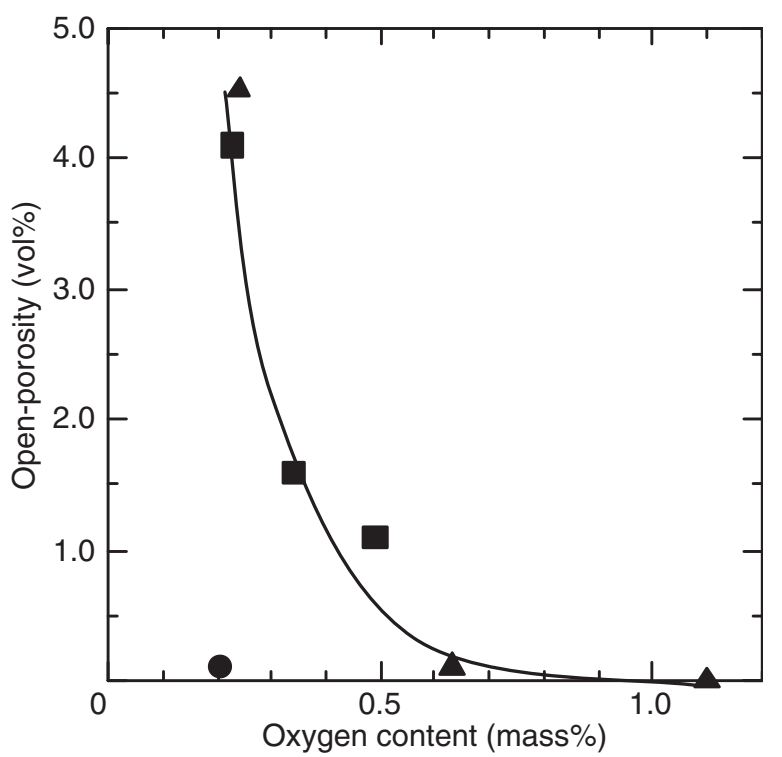

Fig. 2 Relation between open-porosity and oxygen content of HVOF sprayed coatings, ( $\mathbf{\square}$ : SUS316L, $\boldsymbol{\Delta}$ : HastelloyC and $\bullet$ : on-shroud HastelloyC).

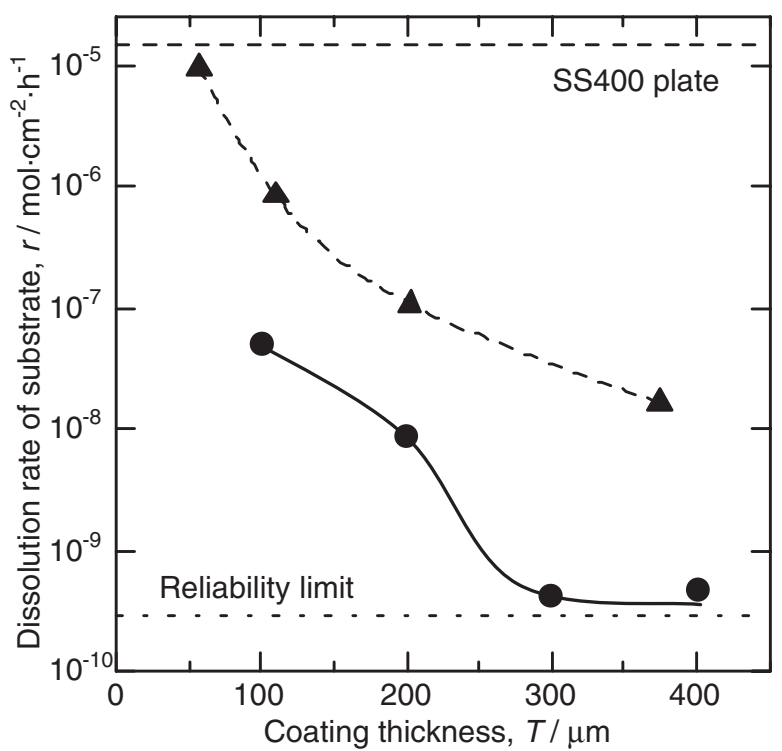

Fig. 3 Dependence of dissolution rate of Fe ion from SS400 substrate on thickness of coating, ( $\mathbf{\Delta}$ : standard HastelloyC and $\mathbf{O}$ : on-shroud HastelloyC).

that a decreasing tendency of the dissolution rate on the coating thickness seemed to be continuous up to about $400 \mu \mathrm{m}$. These results indicated that through-pores remained in this coating. On the other hand, the through-porosity of the on-shroud HastelloyC coating was considered to become zero over $300 \mu \mathrm{m}$ in thickness because the dissolution rate of Fe ion from the substrate through this coating was almost the same value as the reliability limit of this method. The decrease in through-porosity of the coating by attachment of the gas shroud was confirmed also in the cross sectional view of the coated specimens, as shown in Fig. 4. The SUS316L coating (a) had a lot of black spots between the accumulated particles, indicating that it contained the pores or the voids.
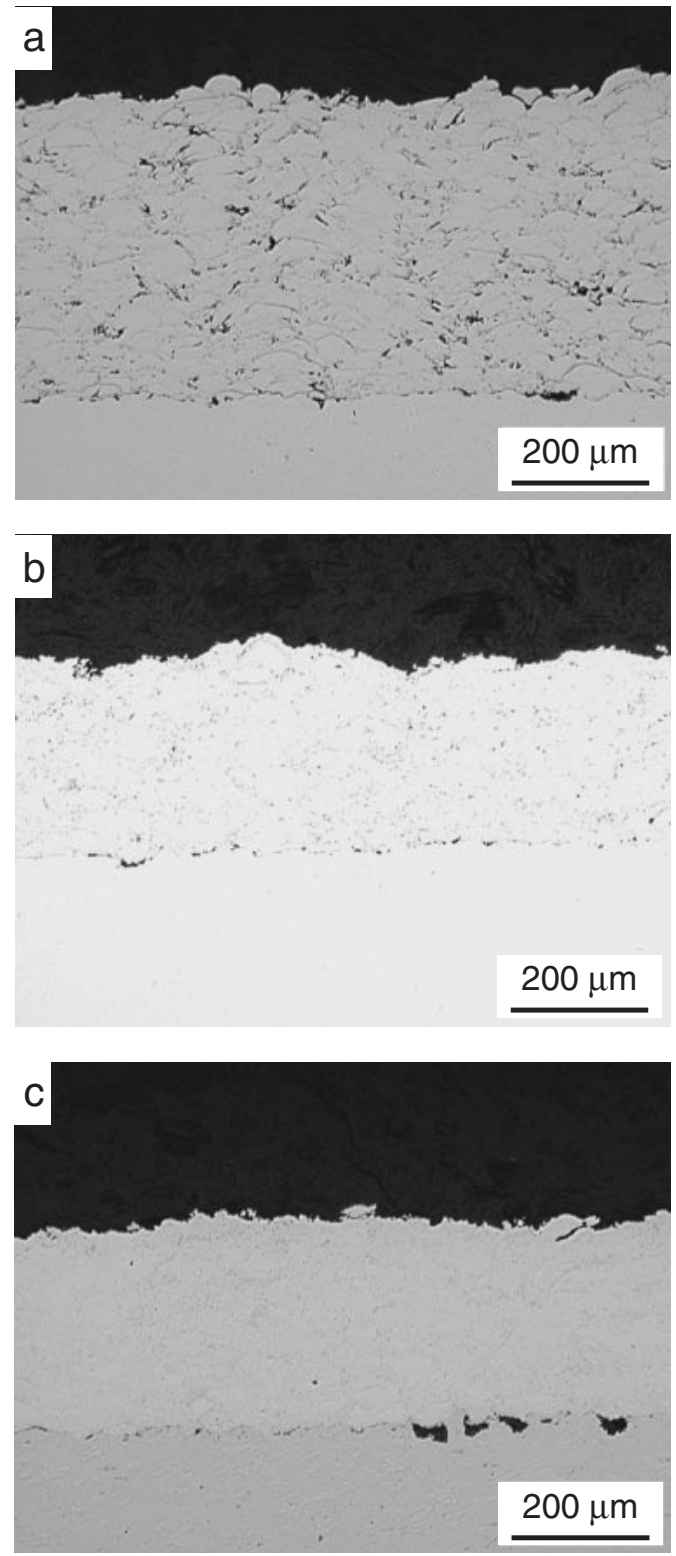

Fig. 4 Optical microscopic images of cross section of HVOF sprayed coatings, (a) SUS316L, (b) HastelloyC and (c) on-shroud HastelloyC.

On the other hand, the HastelloyC coatings seemed to have less or no such pores and voids. The HastelloyC coating without the gas shroud (b) had gray borders surrounding the sprayed particles although such borders became ambiguous as for the on-shroud HastelloyC coating (c). This was due to the decrease of the surface oxide of the particles by using the gas shroud.

The corrosion resistance of the HVOF sprayed coatings is discussed in the following paragraphs. All the specimens for the sample electrodes hereafter were about $400 \mu \mathrm{m}$ in thickness of the coating. By comparing the polarization curves of both the SUS316L and the HastelloyC coatings without the gas shroud in artificial seawater, as seen in Fig. 5, their anodic currents for the coating on the SS400 substrate (solid marks) were larger in the nominal passive region than those for the coatings on the corresponding bulk substrates (hollow marks). This phenomenon is due to corrosion of the substrates caused by the presence of through-pores in 


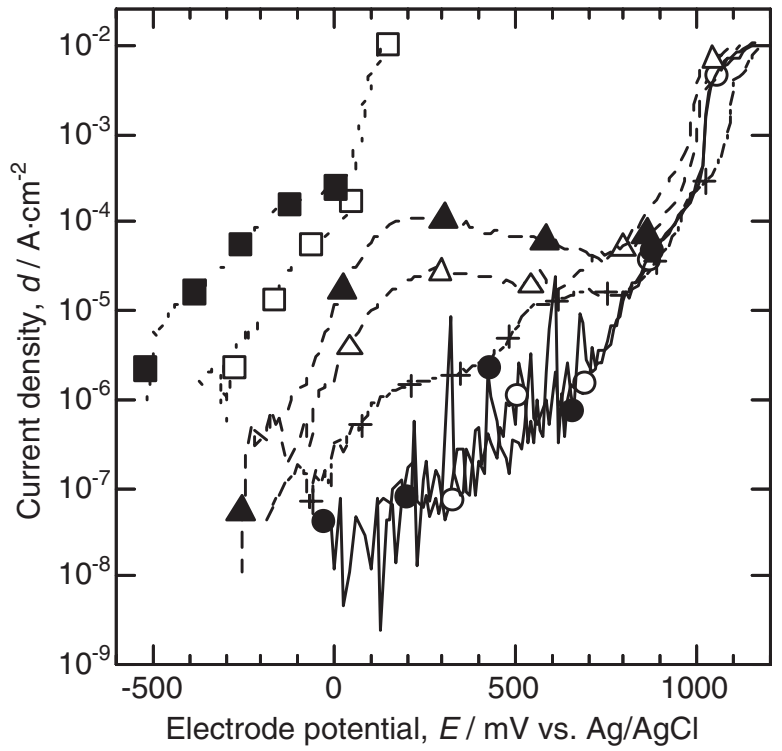

Fig. 5 Polarization curves of HVOF sprayed coatings in deaerated artificial seawater at $300 \mathrm{~K}$, ( $\square$ : SUS316L on SUS316L substrate, $\mathbf{\square}$ : SUS316L on SS400 substrate, $\triangle$ : standard HastelloyC on HastelloyC276 substrate, $\boldsymbol{\Delta}$ : standard HastelloyC on SS400 substrate, $\bigcirc$ : on-shroud HastelloyC on HastelloyC276 substrate, on-shroud HastelloyC on SS400 substrate and +: HastelloyC276 bulk plate).

addition to corrosion of the coatings themselves. Compared to these two coatings, the on-shroud HastelloyC coatings had almost identical curves regardless of the type of substrate. This indicates no occurrence of corrosion of the substrate because of impermeability of the on-shroud HastelloyC coatings.

The electrochemical properties of the coating on the corresponding bulk plate may be regarded as that of the coating itself. As shown in Fig. 5, the pitting potential of the SUS316L coating around $0 \mathrm{~V}$ was much less noble than that of the usual SUS316L bulk material over $500 \mathrm{mV}$, and its anodic current in the passive region was comparatively larger than the bulk material with the value below $1 \mu \mathrm{A} \cdot \mathrm{cm}^{-2}$. These results indicated the poor corrosion resistance of the coating itself. ${ }^{1,9)}$ On the other hand, such pitting corrosion seems not to take place on the HastelloyC coating without the gas shroud while its anodic current was considerably higher, compared to the on-shroud HastelloyC coating. Taking into account the electrochemical characteristics of HastelloyC, i.e. the high resistance against localized corrosion, these results suggested the formation of an oxide on the coating surface in the nominal passive region. In fact, the color of the sample surface changed from less-lustrous gray to brownish green during the anodic polarization test. The on-shroud HastelloyC coating had a lower anodic current in the passive region than both the standard HastelloyC coating and the bulk material of HastelloyC276 while fluctuations of the anodic current appeared with the scanned potential. This phenomenon suggested a kind of local corrosion caused by the microscopic ununiformity of the deposited particles.

Relative corrosion resistance of each coating can be appreciated from the position on the graph of the relation between $V_{d=100}$ and $\Delta V$, as shown in Fig. 6 for the coatings prepared under various spray conditions. Here, $V_{d=100}$

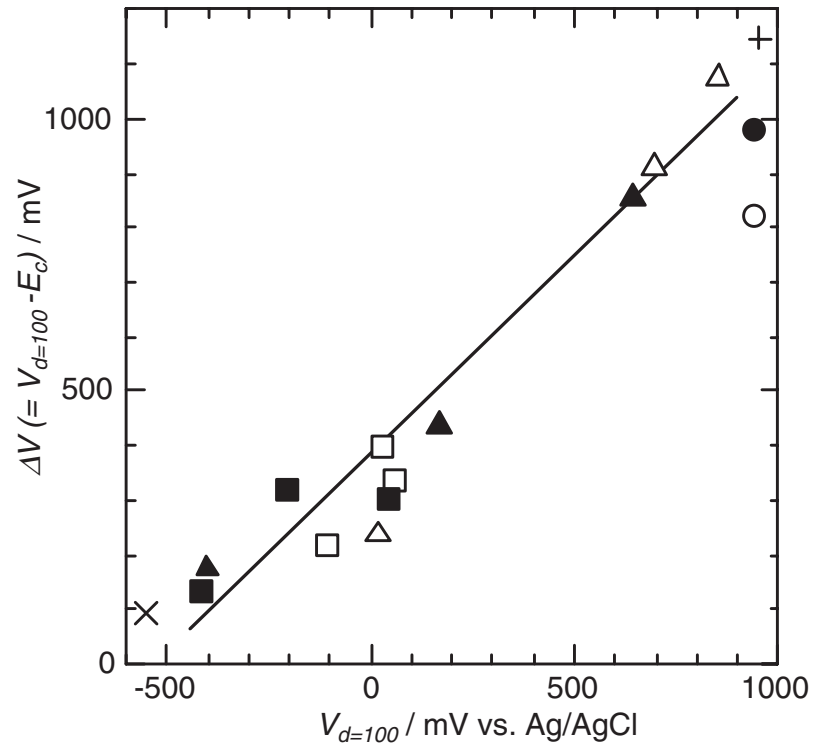

Fig. 6 Relation between $V_{d=100}$ and $\Delta V\left(=V_{d=100}-E_{\mathrm{c}}\right)$, ( $\square$ : SUS316L on SUS316L substrate, $\square$ : SUS316L on SS400 substrate, $\triangle$ : standard HastelloyC on HastelloyC276 substrate, $\mathbf{\Lambda}$ : standard HastelloyC on SS400 substrate, $\bigcirc$ : on-shroud HastelloyC on HastelloyC276 substrate, onshroud HastelloyC on SS400 substrate, +: HastelloyC276 bulk plate and $\mathrm{x}$ : SS400 bulk plate).

designates the electrode potential when the anodic current density reached $100 \mu \mathrm{A} \cdot \mathrm{cm}^{-2}$ on the polarization curve, which is in accordance with the definition of the pitting potential in JIS G 0577-1981. $\Delta V$ is calculated by subtracting corrosion potential from $V_{d=100}$, i.e. $V_{d=100}-E_{\mathrm{c}}$. Higher values in $V_{d=100}$ and $\Delta V$ indicated that the sample has a higher resistance against the local corrosion and a larger passive region, respectively. The SUS316L coatings had the poor corrosion resistance as well as the open-pores with at least $0.2 \mathrm{vol} \%$, leading to be situated in the left and lower positions in this graph regardless of the type of substrate. In Fig. 6, the marks of the HastelloyC coatings prepared without the gas shroud moved toward the right and upper positions with the decrease in open-porosity because the coatings themselves had the comparatively high corrosion resistance. The marks of the on-shroud HastelloyC coatings were situated in almost the same right and upper positions in Fig. 6, resulted from the fact that they possessed both the high corrosion resistance and the zero through-porosity.

As compared in Figs. 7 and 8, the polarization resistance and the corrosion potential of the samples indicated a similar ranking of their values with respect to each other and a similar changing tendency with time except for the on-shroud HastelloyC coatings. In general, the higher corrosion potential and the higher polarization resistance correspond to the higher corrosion resistance. As for the same type of coatings, the difference in their values by the substrates of both the corresponding bulk and the SS400 depended on the degree of through-porosity of the coating. The initial drops in polarization resistance and corrosion potential of the SUS316L and the HastelloyC coatings sprayed without the gas shroud were due to the generation and the progress of corrosion of the substrate, derived from the existence of through-pores. Almost the constant value after these drops indicated the 


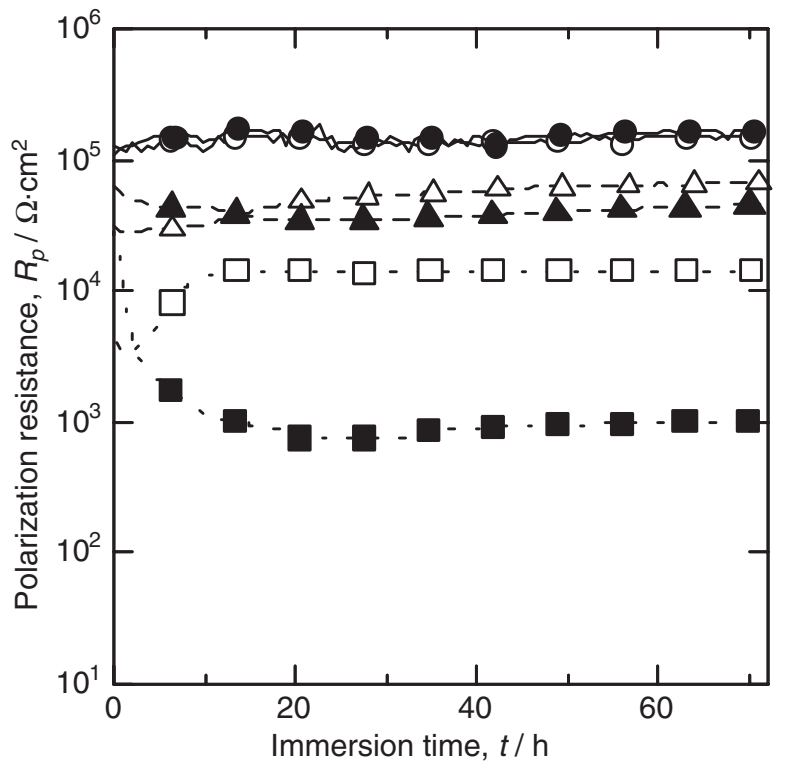

Fig. 7 Variation in polarization resistance of HVOF sprayed coatings with time of immersion in aerated artificial seawater at $300 \mathrm{~K}$, ( $\square$ : SUS316L on SUS316L substrate, $\mathbf{\square}$ : SUS316L on SS400 substrate, $\triangle$ : standard HastelloyC on HastelloyC276 substrate, $\boldsymbol{\Delta}$ : standard HastelloyC on SS400 substrate, $\bigcirc$ : on-shroud HastelloyC on HastelloyC276 substrate and $\mathbf{0}$ on-shroud HastelloyC on SS400 substrate).

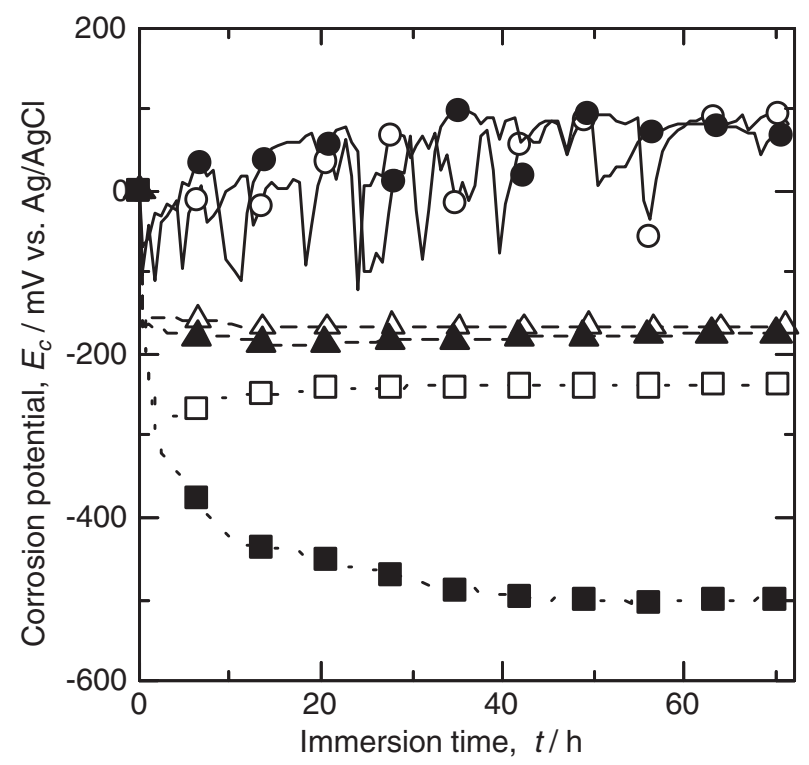

Fig. 8 Variation in corrosion potential of HVOF sprayed coatings with time of immersion in aerated artificial seawater at $300 \mathrm{~K}$, ( $\square$ : SUS316L on SUS316L substrate, $\square$ : SUS316L on SS400 substrate, $\triangle$ : standard HastelloyC on HastelloyC276 substrate, $\mathbf{\Delta}$ : standard HastelloyC on SS400 substrate, $\bigcirc$ : on-shroud HastelloyC on HastelloyC276 substrate and on-shroud HastelloyC on SS400 substrate).

lasting of steady galvanic corrosion. On the other hand, such an initial dropping phenomenon was not observed on the figures in case of the on-shroud HastelloyC coating, implying the absence of through-pores. However, the considerable fluctuation appeared on its corrosion potential while the fluctuation of its polarization resistance was small. The corrosion potential and the polarization resistance depended
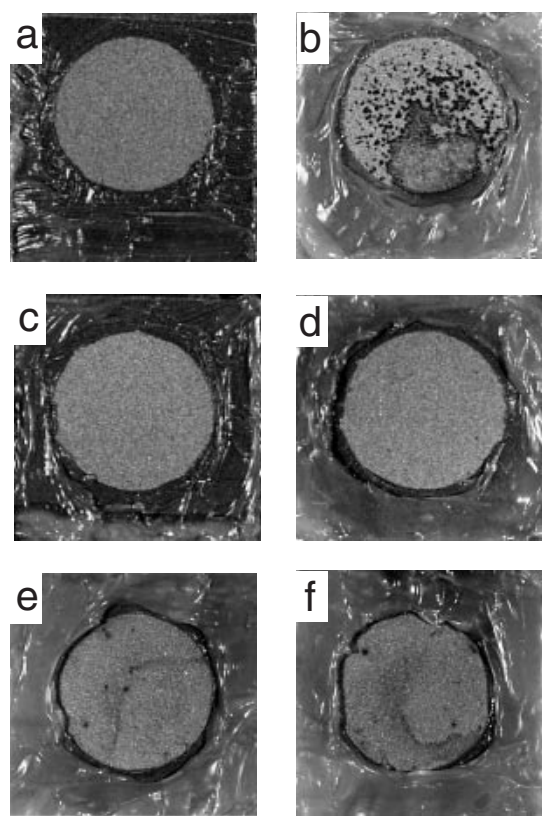

$\overline{10 \mathrm{~mm}}$

Fig. 9 Photographs of HVOF sprayed coatings immersed in aerated artificial seawater at $300 \mathrm{~K}$ for 3 days, (a: SUS316L on SUS316L substrate, b: SUS316L on SS400 substrate, c: standard HastelloyC on HastelloyC276 substrate, d: standard HastelloyC on SS400 substrate, e: on-shroud HastelloyC on HastelloyC276 substrate and f: on-shroud HastelloyC on SS400 substrate).

on the reaction state of corrosion and its comprehensive reaction rate, respectively. Accordingly, a kind of microscopic local corrosion might take place on the on-shroud HastelloyC coatings. The on-going immersion test showed that this phenomenon had not given any effect on the substrate for 4 months.

The corrosion behaviours of the samples were confirmed also by their appearances after immersion in artificial seawater, as shown in Fig. 9. Although only a few small reddish-brown spots could be seen on the surface of the SUS316L coating on the SUS316L bulk substrate (a), many rusts appeared on the surface of the SUS316L coating on the SS400 substrate (b). In the case of the HastelloyC coating sprayed without the gas shroud, the case for the HastelloyC276 bulk substrate (c) kept a thoroughly intact surface while the case for the SS400 substrate (d) had a significantly small black spot on its surface. These results showed that the HVOF sprayed coatings itself had a high corrosion resistance and that corrosion of the substrate occurred because a slight amount of through-pores remained. The on-shroud HastelloyC coatings had some black spots on its surface, regardless of the type of substrate, suggesting the occurrence of the local corrosion, cited above.

Similarly to the appreciation of the relative corrosion resistance of each coating by using the relation between $V_{d=100}$ and $\Delta V$ (see Fig. 6), the corrosion potential and the polarization resistance of the coatings prepared under various spray conditions are compared, as shown in Fig. 10. Higher values in these parameters correspond to a higher corrosion resistance under the just immersed condition without external applying of the electrode potential. Compared to the relation 


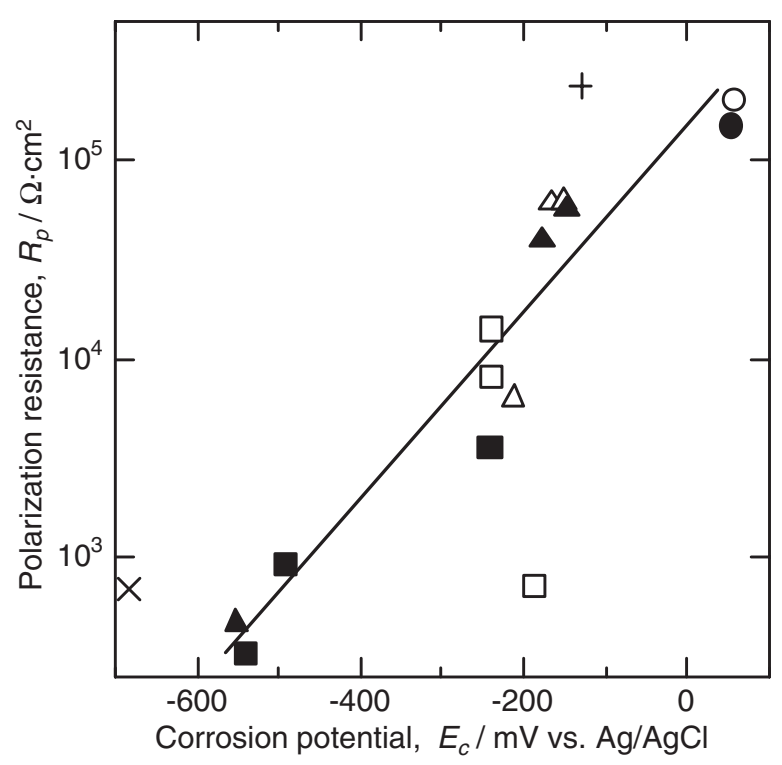

Fig. 10 Relation between corrosion potential and polarization resistance, ( $\square$ : SUS316L on SUS316L substrate, $\mathbf{\square}$ : SUS316L on SS400 substrate, $\triangle$ : standard HastelloyC on HastelloyC276 substrate, $\mathbf{\Delta}$ : standard HastelloyC on SS400 substrate, $\bigcirc$ : on-shroud HastelloyC on HastelloyC276 substrate, on-shroud HastelloyC on SS400 substrate, +: HastelloyC276 bulk plate and $\times$ : SS400 bulk plate).

in Fig. 6, most coatings increased in polarization resistance and/or corrosion potential, particularly as seen in the coatings sprayed on the corresponding bulk substrates. This phenomenon was explained by the following reasons; the impedance measurement was carried out near the equilibrium state, its result was influenced by corrosion of the substrate, derived from the existence of through-pores while the polarization measurement was carried out under the potentio-dynamic condition, the effect of through-porosity on its result became small. This comparison showed clearly that the gas shroud brought about the improvement of the corrosion resistance of the HastelloyC coating. Moreover, its corrosion potential was higher than that of bulk material of HastelloyC276. This result should not be interpreted as that the on-shroud HastelloyC coating had corrosion resistance superior to the HastelloyC 276 bulk material, but that the surface of this bulk material was changed by impacting of alumina grit upon blasting, leading to degradation of its corrosion resistance.

\section{Conclusions}

Adoption of the shroud mechanism by nitrogen gas to HVOF spraying decreases both the through-porosity and the oxide content of the HastelloyC coating. Its through-porosity attained to be zero simultaneously with the oxygen content of 0.2 mass $\%$. Such decreases allowed the corrosion resistance of coated SS400 to increase up to that of the bulk material of HastelloyC276 in seawater. A reaction similar to local corrosion, however, might take place on the surface of this coating. The cause of this phenomenon and its effect on the protective performance of the coating is studied in progress.

\section{REFERENCES}

1) J. Kawakita, T. Fukushima, S. Kuroda and T. Kodama: Proc. ITSC2001, pp. 1137-1142.

2) S. Kuroda, T. Fukushima, J. Kawakita and T. Kodama: Proc. ITSC2002, pp. 819-824.

3) J. Kawakita, S. Kuroda and T. Kodama: Proc. ITSC2002, pp. 681-685.

4) J. Kawakita, S. Kuroda and T. Kodama: Surf. Coat. Technol., 166 (2003) 17-23.

5) T. Fukushima, H. Yamada, J. Kawakita and S. Kuroda: Proc. ITSC2002, pp. 912-917.

6) E. Lugscheider, C. Herbst and L. Zhao: Surf. Coat. Technol. 108 (1998) 16-23.

7) M. Jankovic, J. Mostaghimi and V. Pershin: J. Therm. Spray Technol. 9 (2000) 114-120.

8) D. T. Gawne, T. Xhang and B. Liu: Surf. Coat. Technol. 153 (2002) 138-147.

9) J. Kawakita, T. Fukushima, S. Kuroda and T. Kodama: Corros. Sci., 44 (2002) 2561-2581. 